\title{
The Media and the Chemical Imbalance Theory of Depression
}

\author{
Jonathan Leo • Jeffrey R. Lacasse
}

Published online: 28 November 2007

(C) Springer Science + Business Media, LLC 2007

\begin{abstract}
The cause of mental disorders such as depression remains unknown. However, the idea that neurotransmitter imbalances cause depression is vigorously promoted by pharmaceutical companies and the psychiatric profession at large. We examine media reports referring to this chemical imbalance theory and ask reporters for evidence supporting their claims. We then report and critique the scientific papers and other confirming evidence offered in response to our questions. Responses were received from multiple sources, including practicing psychiatrists, clients, and a major pharmaceutical company. The evidence offered was not compelling, and several of the cited sources flatly stated that the proposed theory of serotonin imbalance was known to be incorrect. The media can play a positive role in mental health reporting by ensuring that the information reported is congruent with the peer-reviewed scientific literature.
\end{abstract}

\section{Keywords Depression ·SSRI $\cdot$ Serotonin}

In the world of American popular culture, the current view of mental illness depicts someone walking down the street, and everything is fine, life is good. Then all of a sudden, out of the blue, a chemical imbalance emerges. At the root of every twisted thought lurks a twisted molecule - so the thinking goes. A large part of the credit for this belief certainly goes to the pharmaceutical companies, yet they are not the only source of information on mental health issues. For many people, another major source of information is the mainstream

\footnotetext{
J. Leo $(\square)$

Lincoln Memorial University, 6965 Cumberland Gap Parkway,

Harrogate 37752, USA

e-mail: Jonathan.leo@lmunet.edu

J. R. Lacasse

Florida State University (FSU),

Tallahassee, USA
}

press, which often alludes to the chemical imbalance theory as if it were a proven scientific fact. In an effort to determine what evidence the press uses to support their statements about the chemical imbalance theory of depression, we attempted to engage the media in a conversation to learn more about what lies behind statements such as: "Mental illnesses are simply chemical imbalances." In support of their statements, several members of the press did not provide any citations, while some referred us to interesting articles about serotonin research, but not a single citation could be considered direct proof of an underlying chemical imbalance as the cause of depression. (The term 'mental illness' is used throughout this article due to the fact that it often appears in media accounts. The more technical term, "mental disorder," is considered the more correct term within modern psychiatric literature. There is ongoing debate regarding the definition, utility, and scientific validity of "mental disorder.")

\section{The Chemical Imbalance Theory}

The idea that depression results from a chemical imbalance in the brain was first proposed in the late 1950s and early 1960 s by several different scientists. The focus was initially on the neurotransmitter norepinephrine, but by the mid 1960s the focus had shifted to serotonin, another transmitter, which ultimately led to the development of the Selective Serotonin Reuptake Inhibitors (SSRI) such as Prozac and Paxil. At the time, there were several lines of convergent evidence but the strongest evidence came from the observation that the administration of pharmacological agents acting on the brain's monoamine system could either increase or decrease mood levels. For instance, it had been known for several years that, at least in some people, the drug reserpine could lead to a depressed state. Subsequent studies in rabbits showed that reserpine causes reductions in serotonin. Thus, putting the two facts together, deductive reasoning suggested that depression 
could be the result of too little serotonin. In essence, as summarized in a table in one of their early papers, the researchers observed that mood could be artificially manipulated with drugs - those which raised monoamine levels improved mood, while those which lowered amine levels led to depression, but it remained to be seen if naturally occurring fluctuations in neurotransmitter levels were responsible for, or caused, the ebb and flow of mood levels. In the words of Schildkraut, one of the early proponents of the theory: "At best, drug-induced affective disturbances can only be considered models of the natural disorders, while it remains to be demonstrated that the behavioral changes produced by these drugs have any relation to naturally occurring biochemical abnormalities which might be associated with the illness" (1965). Like any good hypothesis, the "chemical imbalance theory," as it has come to be called, provided a springboard for a tremendous amount of research, and subsequently transformed two important sectors of the health care system - the psychiatry profession and the pharmaceutical companies.

For the psychiatry profession, the theory was a major driving force behind the rise of biological psychiatrists within the profession, and moved the profession from one involved in talking to patients about everyday problems, to a profession that was treating their patients' organic diseases-just as the internists were treating diabetics suffering from insulin shortages, the psychiatrists were now treating depressed patients suffering from serotonin shortages. Depression was no longer seen as just a natural response to stress, there was now an underlying biological factor which was the cause of the depression.

The theory also transformed the pharmaceutical companies. For instance, Sertraline (Zoloft) was the sixth bestselling medication in the US in 2004 , with over $\$ 3$ billion in sales likely due, at least in part, to the widely disseminated advertising campaign starring Zoloft's miserably depressed, and presumably serotonin deficient, ovoid creature. No matter how you look at the data, the figures are impressive: In July of 2007, a government study found that antidepressants are the most prescribed drugs in the US; during the past 6 years patients spent $\$ 123$ billion on psychotropic drugs; in 2005, doctors wrote 31 million prescriptions for antidepressants; and in 2004, the pharmaceutical companies spent $\$ 1.5$ billion promoting antidepressants. Prior to the idea of chemical imbalances, the pharmaceutical companies had advertised their wares as tonics which could ease people through the ups and downs of normal, everyday existence. For instance, in the 1950s Butisol was advertised as "the 'daytime sedative' for everyday emotional stress" and in the 1970s Valium was advertised for the "unremitting buildup of everyday emotional stress resulting in disabling tension."

As in many discussions about scientific concepts, there is often confusion over the terms "causation" and "correlation." The difference between the two terms may seem trivial at first glance, but the definitions have enormous implications for any discussion about the relationship between psychology and biology. Evidence of biological changes correlating with environmental stressors is vastly different from evidence that mental illnesses are "caused" by biological deficits. For instance, a correlation between abnormal behavior and altered brain activity does not necessarily imply that the behavior was caused by a brain defect. There is a growing body of research showing that biological changes could be due to environmental stimuli. Take the hippocampus, a brain structure important in learning and memory: It has been shown that chronic psychological stress can reduce the size of the hippocampus, and that, conversely, 3-D spatial relationship training leads to enlargement of the hippocampus. As another example, a recent meta-analysis found that $69 \%$ of the female patients diagnosed as psychotic in over 70 studies had reported either physical or sexual abuse. It is entirely possible that the women in the study developed a chemical imbalance, but it would be difficult to determine if their conditions resulted from genetic defects or environmental stressors.

In addition to psychological stress, physical stress can lead to depression. A recent study of ex-NFL players documents a correlation between numbers of concussions and depression later in life. As this example makes clear, even if some players have a genetic predisposition to traumatic brain injury, if you take away the environmental insult (in this case getting knocked in the head), there would be no disease. Regardless of any genetic defect, any subsequent deficits are $100 \%$ preventable by avoiding the football field.

As an example of someone who is well placed to understand the theory, along with the difference between correlation and causation, take Nancy Andreasen, a prominent biological psychiatrist, who sums up biological psychiatry's view of mental illness in her book, The Broken Brain: "these diseases are caused [italics added] principally by biological factors and most of these reside in the brain." Andreasen is referring here to "causation," not just "correlations." In the United States, the diagnosis of depression doubled between 1991 and 2000, coinciding with the introduction of the SSRI medications. These medications were not marketed by explaining the problematic correlational data; instead, they were sold as remedying the chemical imbalance that caused depression.

As another example of how subtle nuances in semantics have important implications for our understanding of mental illness, take the following statement from Diane Patrick, married to the governor of Massachusetts, Deval Patrick. Following treatment for her depression, she gave a press briefing in 2007 in which she said, "I've done a little reading in the time I've had. And what I know is that depression is really a chemical imbalance, which is triggered by systemic stress [italics added]." Patrick, whose carefully chosen words also indicate that she understands the difference between causation and correlation, has a dramatically different view 
of mental illness compared to Andreasen's view that biological alterations are the cause of mental illness; for Patrick, the chemical imbalance is caused by prolonged stress. And these two different scenarios have vastly different implications for the use of drugs.

With the advent of the chemical imbalance theory, the companies were no longer just providing soothing tonics, they were now providing medications to treat diseases, as exemplified by an early SSRI advertisement stating: "When serotonin is in short supply, you may suffer from depression." The wording here is all-important. The advertisement takes a correlation between serotonin shortage and psychological stress - and even this is highly questionable and unverifiable in any individual case - and makes a leap of faith to the conclusion that depression is caused by a serotonin imbalance, not that psychological stress impacts the serotonin system. And the marketing did not stop with depression; eventually we were told that whatever our problems might be, whether anxiety, excessive shyness, depression, or the inability to pay attention, the underlying cause was a faulty transmitter level which could be rectified with a pill. A 2005 survey from the Harvard School of Public Health reported that nearly half of all Americans will at some point develop a mental illness, presumably from a chemical imbalance, with $29 \%$ developing an anxiety disorder and $20 \%$ a mood disorder.

Substances used to rectify a chemical imbalance can be called medications and are consumed by patients, while substances that are used to "take the edge off" are typically considered street drugs and are consumed by users or addicts. But is this line between legal and illegal really that straightforward? When we assign a mood-altering drug to either one of these two categories are we basing this on science or a social agenda? There are a whole host of substances, some of which are legal-alcohol, valium, etc.-and some of which are illegal - marijuana, heroin, etc.- that humans have taken for years to ease anxiety and depression. For an example of how artificial these categories are, take the case of Ricky Williams, the star running back for the Miami Dolphins who has been diagnosed with Social Anxiety Disorder. In 2002, People magazine, in a statement closely resembling a pharmaceutical company advertisement, referred to Williams' disease as a "depression-like chemical imbalance that affects roughly three million Americans." For several years, Williams was paid by Glaxo to promote Paxil, an SSRI. During his career, Williams was in and out of trouble with the NFL and tested positive for marijuana on several occasions. While his marijuana use was frowned upon, his use of Paxil was considered acceptable. One was a medication supposed to treat a chemical imbalance, while the other was a drug signaling a lack of willpower. In 2004, Williams' job with Glaxo came to a sudden halt when he stated that marijuana was ten times better than Paxil. Williams did not get into trouble for saying that another drug was better than the sponsor's drug (as, say, a Coke representative would have had he admitted he really preferred Pepsi). What actually got Williams into hot water was that he put his sponsor's drug in the same category as an illicit drug. The NFL, the psychiatry profession, the pharmaceutical companies, and People magazine all operate under an assumption that the line between legal and illegal is simple and concrete. Williams' acknowledgement that it is complicated and uncertain posed a dilemma for the pharmaceutical companies, who were footing his bill, and he was let go.

In addition to the correlation-causation issue another area of confusion is research into the mechanism of SSRI action. The thinking goes that, because the SSRIs act on the serotonin system, their efficacy must be due to an existing imbalance of serotonin. This line of reasoning is logically problematic as LSD also acts to increase serotonin levels, and we do not make similar claims about LSD-induced hallucinations only occurring in chemically deficient individuals. Or take Glaxo's new over-the-counter diet drug, Alli, which helps dieters lose weight-no matter what the person weighs. Alli's actions are not limited to overweight people, but will lead to weight loss even in thin people, just as the SSRIs have an effect on everybody, not just those who are depressed.

In a 1989 memorandum, the head of Pfizer's French headquarters commented on the wording of the French packaging label for Sertraline (Zoloft): "But considering the fact that the monoaminergic hypothesis of depression is not unique and that some authors have even stated a hyperfunctioning of the monoaminergic pathway, it seems therefore preferable not to write anything about etiopathogeny of depression, and to describe only the actions [sic] of Sertraline." The importance of this document stems from the fact that, as early as 1989, even Pfizer acknowledged that talking about a drug's action is not the same thing as talking about the etiology of depression. In other words, evidence of a drug's action is not evidence of etiology.

Psilocybin is the active ingredient in mushrooms, an illegal drug which causes hallucinations. Some have suggested it was the inspiration for Lewis Carroll's Alice in Wonderland. Recently, researchers at Johns Hopkins ran a controlled clinical trial investigating the antidepressant effects of psilocybin and, according to their results, 79\% reported moderately or greatly increased levels of life satisfaction. Presumably, few would argue that psilocybin's actions on the brain or that the production of Alice in Wonderland was the result of a chemical imbalance. As this example illustrates, one cannot simply assert that the claimed efficacy of mental health drugs necessarily equates to a chemical imbalance. If we apply the same type of logic that we use to support the chemical theory of depression to our thinking about mushrooms then mushrooms should be categorized as a medication. 
Some professionals have pointed out that from a practical point of view, the validity of the theory is not really that important. After all, as long as the medication works, does it really matter whether we know what the mechanism is? This argument would be reasonable if the advertisements were limited to the medication's effects, however a major portion of the pharmaceutical company advertising campaigns has focused on the mechanism of depression. Take this advertisement for Lexapro: "In people with depression and anxiety, there is an imbalance of serotonin." If you took away the chemical imbalance theory and only advertised the SSRI's effects, the marketing departments would be left with little. Without a chemical imbalance, the SSRIs are just another group of mood-altering drugs.

In spite of the enormous amount of money and time that has been spent in the quest to confirm the chemical imbalance theory, direct proof has never materialized. Moreover, during the past several decades, a significant amount of evidence has accumulated which calls the theory's validity into question. Perhaps most problematic for the theory, over the past two decades, multiple studies have shown that the once presumed efficacy of the SSRIs, the foundation upon which the theory was built, is not nearly as robust as once thought. In 2002, Irving Kirsch and colleagues at the University of Connecticut, using the Freedom of Information Act, gained access to all clinical trials of antidepressants submitted to the Food and Drug Administration (FDA) by the pharmaceutical companies as part of the medication approval process. When the published and unpublished trials were pooled, the placebo duplicated about $80 \%$ of the antidepressant response - in Prozac's case it was $89 \%$. In other words, for every ten people who take an SSRI, only one or two people are truly receiving a benefit from the medication - a fact acknowledged even by the SSRI proponents. The obvious question is: What about the eight or nine people getting no benefits from the medication but now put at risk for the medication's side effects? Given these and other problems with the chemical imbalance theory, there are now few researchers willing to step up and mount a full-scale defense of it.

In an earlier paper we examined the differences in how the theory is portrayed in the scientific literature versus the pharmaceutical company advertising, and reported that the incongruence between the scientific literature and the claims made in the SSRI advertisements is remarkable, and possibly unparalleled. Take this comparison as just one example: The American Psychiatric Press Textbook of Clinical Psychiatry addresses serotonin deficiency as an unconfirmed hypothesis, stating, "Additional experience has not confirmed the monoamine depletion hypothesis," while a recent advertisement for Celexa states: "Celexa helps to restore the brain's chemical balance by restoring the supply of a chemical messenger in the brain called serotonin." The textbook and the advertisement have very different positions on the theory.
Although silence from critics is certainly not confirmation of our claims, numerous scientists and psychiatrists have responded either in interviews, or in published lettersto-the-editor about our thesis, and not a single one has disagreed. In one interview about our article, the head of the FDA Psychopharmacology Advisory Committee stated that the chemical imbalance theory is a "useful metaphor" but not one that he uses with his own patients.

There is then a dilemma for the majority of the population who do not read psychiatry journals and who are much more likely to learn about scientific concepts from the mainstream media, either from magazines, such as Time and Newsweek, or from newspapers, such as the New York Times or LA Times. To determine the evidence behind the media's claims about chemical imbalances, for approximately 1 year we performed weekly Internet searches of the media for "chemical imbalances" and sent e-mails to the authors asking them for the evidence they were basing their statements on.

\section{Evidence-based Reporting?}

In an article for the Sacramento Bee (3/9/07), about how to handle teenagers with depression, the author states: "Act promptly and accept that they may have a chemical imbalance [italics added] or need help with coping skills." In reply to our questions, the author mentioned that: psychiatrists would be the best people to talk with about chemical imbalances; mental illnesses have been linked to chemical imbalances; psychiatrists are trained to figure this out through a variety of tests; and that "numerous studies have been done" and "the research is definitely available." We pointed out to her that, if there are "numerous studies" which are "definitely available," then it should be relatively easy to cite at least one article. She did not reply. We also mailed a copy of our e-mails to an editor at the Sacramento Bee. On March 29, 2007, another newspaper published a copy of her article with the comment about chemical imbalances removed. The edited article appears at: http:// www.contracostatimes.com/teens/ci_5513238.

In ScienceNOW Daily News (10/18/06), an article discussed new research suggesting that Prozac increases bone density. In the article, the author stated, "Researchers believe depression results from too little of the neurotransmitter serotonin bathing certain neurons." In an e-mail, she replied that she did not go back to find the original references and that it would be difficult "to start from scratch every time I wrote a news article." She did not supply any references other than to refer us to the original research paper on bone density, and told us that we were free to check their references. The initial research article also mentions the chemical imbalance theory and cites a 
1991 article by Mann which appeared in a Neuropsychopharmacology journal supplement.

In the New York Times (12/31/06), Michael Kimmelman wrote about the life and work of Joseph Schildkraut, one of the founders of the chemical theory of depression. The Times reporter stated, "A groundbreaking paper that he published in 1965 suggested that naturally-occurring chemical imbalances in the brain must account for mood swings, which pharmaceuticals could correct, a hypothesis that proved to be right [italics added]." E-mails to the author requesting a citation to support his statement went unanswered.

In another New York Times article (6/19/07), "On the Horizon, Personalized Depression Drugs," Richard Friedman, the chairman of Psychopharmacology at the Weill Cornell Medical College, stated: "For example, some depressed patients who have abnormally low levels of serotonin respond to SSRIs, which relieve depression, in part, by flooding the brain with serotonin." For his evidence he supplied a 2000 paper by Nestler titled, "Neurobiology of Depression," which focuses on the hypothalamic pituitary system but not on serotonin.

In the Georgetown News (3/20/07), the author of "Depression: The Silent Sickness," who had been diagnosed and treated for depression, stated: "When I was first diagnosed with depression, or a chemical imbalance..." In an e-mail, she stated that her source was her primary care doctor who told her that she made too much adrenaline.

An anonymously-written article in the Economist (3/5/ 07), defined serotonin as follows: "This molecule is a neurotransmitter (a chemical messenger between nerve cells) and one symptom of depression is low levels of it." The Economist article was generated from a press release announcing the results of a study from the University of Bristol. The press release stated: "The lack of serotonin in the brain is thought to cause depression in people." The author of the press release has not replied to our inquiries.

In the Kane County Chronicle (4/28/07), an article appeared about suicide with several quotes from Mari Wittum of Suicide Prevention Services. According to the reporter, in a talk about reducing suicide in teenagers, Wittum discussed the causes of mental illness and said that the "impetus often could be a chemical imbalance or hereditary illness." The author forwarded the request to Wittum who provided two websites, one from her own organization and one from National Alliance on Mental Illness (NAMI). She did not provide any specific citations but said that chemical imbalances were one reason people committed suicide. She also said that her interest lies in the clinical side of things.

In the Vancouver Sun (2/24/07), an article quoted several psychiatrists on the subject of mental illness, one of whom makes this statement about the antidepressants: "they seek to correct chemical imbalances in the brain usually caused by a genetic predisposition to them." When asked about the references, the author referred us to the psychiatrist quoted in the article, who in turn supplied the following citations. The first, a 1991 study titled, "Measures of Serotonin Function Are Reduced in a State Dependent Manner" by Upadhyaya and colleagues in the Journal of Affective Disorders (Vol. 21), showed that in depressed patients there is an alteration of receptor numbers. However, as in all these types of studies, and as pointed out by all the researchers, it is not clear whether these changes are signs of an organic defect causing the depression, or if environmental stressors have caused the changes in the brain. We are not aware of any replications of this study in the 15 years since it was published. Presumably, if it had been replicated, the subsequent studies would have been cited.

The second citation was a highly theoretical article, written in 2006, about serotonergic vulnerability by Jans and colleagues in Molecular Psychiatry (Vol. 9), in which the authors state, "By now, however, the model of a deficit in 5-HTergic neurotransmission being primary in the causation of depressive disorders, and predictive of therapeutic response to drugs enhancing 5-HTergic neurotransmission has become obsolete: not all depressed patients present with 5-HT abnormalities, not all patients benefit from drugs enhancing 5-HTergic neurotransmission, and several drugs that are devoid of major effects on 5-HTergic neurotransmission are known to be effective antidepressants." The article does present a hypothesis about chemical imbalances: Just as you can measure and subsequently plot a graph of a physical trait, such as height, you can theoretically do the same with serotonin function. Therefore, just as with height and weight, there must be some portion of the population on the left-hand side of the graph. Rather than attribute this to normal biological variation, the authors speculate that the subjects on the far side of the curve have "theoretical serotonergic vulnerability" which makes them more susceptible to environmental stress. Interestingly, the authors point out that more women than men have this defect. The article also ends with the rather surprising idea that we will eventually be able to identify these people on the far side of the curve and pre-treat them for environmental stressors. In short, the article is interesting but provides no confirmatory proof for the chemical imbalance theory.

The third citation was a 1999 article by Porter in The Journal of Psychopharmacology (Vol. 146) which is not about chemical imbalance as a cause of depression, but is about how the SSRIs act on the serotonin receptor. As mentioned earlier, few, if any, scientists would disagree with the fact that the SSRIs act on the serotonin receptor, but as pointed out by the original proponents of the serotonin theory, this does not provide evidence that depression is caused by low serotonin. 
In the Philadelphia Inquirer, Alexis Sohrakoff wrote that "mental illnesses are simply chemical imbalances." In an email, she supplied the following references. The first was a 2000 article by Zubieta and colleagues in the American Journal of Psychiatry (Vol. 200) about transporter binding in asymptomatic bipolar I disorder. The article noted a difference in the number of monoaminergic synaptic terminals between sixteen patients diagnosed with bipolar compared with normal controls. The study has not been replicated to the extent that scientists can say that they have found a marker for bipolar patients.

Sohrakoff also supplied a statement, made by the president of the Society of Neuroscience, which was part of a request made to a congressional panel for more funding: "mental illnesses were due to a chemical imbalance." When the problems with these citations were pointed out to the author, she replied, "I did not conduct an extensive literature search, as I assumed that if an individual such as the president of the Society of Neuroscience, among others, stated that a mental illness represents a chemical imbalance, there must be some evidence to that fact."

In the Helena Independent Record (6/10/07), a reporter wrote, "Sometimes it's [depression] the result of a chemical imbalance, such as serotonin levels. Fix the imbalance, fix the depression." As evidence, the author supplied a link to a web page at the National Institutes of Mental Health (NIMH), which in turn said, "Researchers believe that both depression and suicidal behavior can be linked to decreased serotonin in the brain. Low levels of a serotonin metabolite, 5-HIAA, have been detected in cerebral spinal fluid in persons who have attempted suicide, as well as by postmortem studies examining certain brain regions of suicide victims."

To dig deeper, we contacted NIMH and asked for their references. As a citation for their statement, they supplied a 2003 review of the genetics of the serotonergic system in suicidal behavior by Arango and colleagues in the Journal of Psychiatric Research (Vol. 37). The review discussed the research of four genetic polymorphisms involved in the serotonin pathway: one polymorphism had some preliminary results suggesting a correlation between it and suicide, while the other three were not promising. In their conclusions the authors state: "At present there is no identified 'suicide' gene, nor is any one gene likely ever to be found causal to suicidal behavior." The authors then go on to speculate about future possibilities. Besides citing this one review article, NIMH also suggested doing a Pub Med search. We replied to NIMH that we were not interested in the thousands of general citations on Pub Med, but, instead, were interested in the specific evidence that they, NIMH, were using to support their statements. We have not heard back from NIMH.

In the Pittsburgh Tribune Review (4/2/07), Noelle Creamer wrote, "It's not a personal deficit, but something that needs to be looked at as a chemical imbalance." This quote was attributed to a psychiatric nurse practitioner. The author did not respond to e-mails, and the nurse's e-mail was not available.

The Bradenton Herald (3/24/07), published an article entitled, "Seniors Sought for Depression Study." The primary source for the article was Dr. Andrew Cutler, the director of the Florida Clinical Research Center, who is extensively quoted and referred to by the reporter. "True depression," Cutler says, "has its roots in a chemical imbalance in the brain." Neither the reporter nor Dr. Cutler replied to e-mails.

In the Gilroy Dispatch (4/24/07), the author wrote, "A patient with a chemical imbalance that leads to depression likely receives entirely different insurance coverage than a patient with a chemical imbalance that leads to hyperthyroidism receives." The author has not responded.

In The Gazette (4/17/07), an article claimed, "Depression is a medical illness caused by a chemical imbalance in the brain." The author has not replied. We also posted our inquiry as a comment to the article.

\section{Reporters Covering Mental Illness}

Prior to our efforts to solicit evidence from reporters, there were two well-known cases of nationally-recognized reporters discussing chemical imbalances. Following Tom Cruise's controversial remarks in 2005, CNN medical reporter Sanjay Gupta attempted to counter Cruise by using brain scans to demonstrate that chemical imbalances were in fact, real. On the air, he showed PET scans of depressed individuals, remarking that: "When you don't have enough neurotransmitters firing, making the connections, your brain doesn't activate in the area that it should. And you can see what a normal brain should look like. That is an objective measure..."

Psychiatric Times, a trade journal, published an article where Gupta defended his remarks, stating that he was just trying to demonstrate that there is objective research in mental health. But his statement and the display of PET scans probably misled the overwhelming majority of CNN's audience who are not familiar with the legitimate use of this technology by the psychiatry profession. That use is for research purposes, and not for identifying depressed individuals. Although a PET scan might someday accurately show how brains differ in terms of serotonin receptors, it is an entirely different matter to use a scan to document how the brain reached that state. An X-ray can accurately identify a broken bone, but it can't tell you whether the bone was broken in a car accident or a football game. The same is true of a PET scan - it cannot tell you whether its findings are due to stress, thinking patterns, social isolation, trauma, or whether they are even clinically significant. A neuroimaging expert cannot look at a PET scan of an individual and make 
a psychiatric diagnosis. The use of technology to imagine the brain is impressive and promising, but it does not provide easy answers to the difficult questions that must be asked of the chemical imbalance theory.

Another instructive example was the June 27, 2005 Today show interview, where psychiatrists Dr. Joseph Glenmullen and Dr. Steven Sharfstein squared off on the issue of chemical imbalances. Glenmullen, a clinical instructor in psychiatry at Harvard Medical School and a critic of the theory, stated that the chemical imbalance theory was fallacious, but explained that he still prescribed the medications when he felt they would be helpful. Sharfstein, president of the American Psychiatric Association, found this point of view to be "total nonsense," yet he also stated that there was a constructive debate going on in psychiatry about these topics. The idea that there is an ongoing debate sounds nice, but is it true? The Diagnostic and Statistical Manual of Mental Disorders, which almost all psychiatrists use to diagnose and treat their patients, clearly states that the cause of depression and anxiety is unknown. In addition, for well-accepted diseases, such statements are not debated, but settled through scientific research. For instance, if a critic were to argue that neurosyphillis was not a disease, an infectious disease specialist could locate and cite the paper which proves this incorrect-and the debate would be over. Most interestingly, while Sharfstein maintained the idea that the chemical imbalance theory was a fact, Today's Katie Couric did not ask him what evidence he was referring toobviously we would have asked.

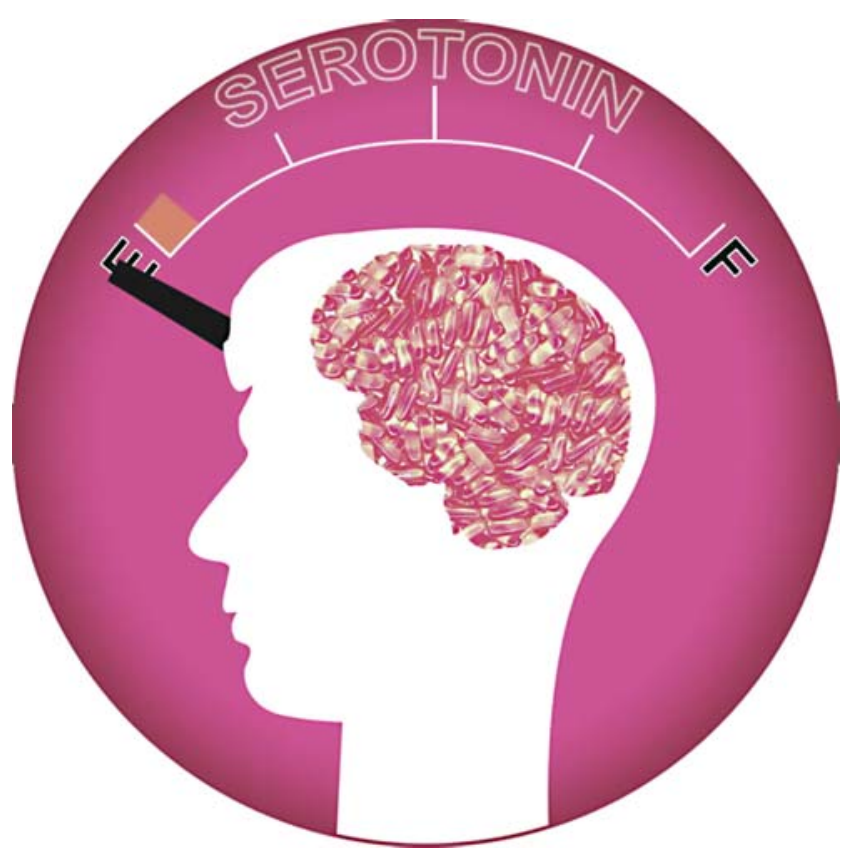

\section{The Pharmaceutical Companies and Chemical Imbalances}

Considering the media's inability, or unwillingness, to cite evidence in support of their own statements, can the same group really be expected to go one step further and actively investigate these issues? The solution is not simply for the media to modify, or tone down, their own statements about the chemical imbalance theory, but is for them to take a more analytical approach with those who promote the chemical theory as ineluctable truth. In other words, rather than us questioning the media, shouldn't the media be doing the questioning? It's almost as if these reporters are blinded by the term, "peer reviewed," and operate under the mistaken assumption that the words are some sort of stamp declaring that the results are unquestionable and that they can check their skeptical radars at the door when given a press-release mentioning a peer-reviewed article.

While the press continues to mention chemical imbalances, as mentioned earlier, academic psychiatrists have not disagreed with the idea that the SSRI advertisements are an inaccurate portrayal of the chemical imbalance theory, but at least one pharmaceutical company has. In response to our article on the SSRI advertisements, a Pfizer spokesman mentioned to a reporter that: "There is considerable scientific literature supporting the widespread belief among scientists and physicians that an inadequate level of serotonin in the neuronal synapses of the brain is at least one of the causes of depression." The spokesperson also provided the reporter with the following citations as evidence in support of the chemical imbalance theory: two textbook chapters and a review paper.

Pfizer's first citation, a chapter from The American Psychiatric Textbook of Psychopharmacology (1998), was authored by Gary Tollefson, the head of neuroscience at Eli Lilly, and Jerold Rosenbaum, a member of the premarketing advisory committee for Prozac. The chapter provides little in the way of a discussion about the mechanism of depression, and has little bearing on the chemical imbalance theory, but the chapter does provide some insight into the commercial success of the SSRIs. The chapter is mainly concerned with the clinical use of the SSRIs. Two major concerns for a clinician are efficacy and side effects, not just for the SSRIs but for any drug. Clinicians hearing a pharmaceutical company-sponsored presentation will likely understand that the drug will be portrayed in the best light possible, with the side effects being downplayed and the efficacy exaggerated - this assumes that clinicians are a somewhat representative sample of the general population, $93 \%$ of whom recently reported being skeptical of pharmaceutical company claims. However, what about textbook chapters, which are traditionally thought of as providing a more realistic presenta- 
tion? Should clinicians maintain that same healthy dose of skepticism when reading textbook chapters as they do when hearing a pitch from a salesman?

Regarding efficacy, Tollefson and Rosenbaum state: "A plethora of placebo-controlled, double blind trials have established the clear superiority of the SSRI's over placebo" (p. 224). However, this statement seems overly enthusiastic regarding the SSRIs. Even when Eli Lilly first submitted its request for approval from the FDA for Prozac the evidence was marginal, at best. For instance, out of eight separate studies combined to make up three trials submitted to the FDA, only four of the studies found a statistically significant advantage of Prozac over placebo. One of the studies lasted 4 weeks and included 11 people. Several of the studies were pooled together so that two of the three trials were significant.

In support of their statement about efficacy, Tollefson and Rosenbaum cite a 1992 review by Kasper published in an SSRI manufacturer-sponsored (Fluvoxamine) supplement to the journal, Drugs (Vol. 43). Interestingly, as David Healy documents in Let Them Eat Prozac, Kasper had previously allowed pharmaceutical companies to publish papers written by ghostwriters under his name. In 1999, the pharmaceutical company, Pierre Fabre, presented a prewritten scientific paper to Healy, asking if it could designate him as the author before submitting it to a journal. Because Healy wanted to make changes to the article that the company did not agree with, Pierre Fabre moved on. The article was subsequently published with Kasper as the author.

Regarding their reporting of side effects, when it comes to the potential link between the SSRIs and suicide, Tollefson and Rosenbaum state, "that no evidence indicated that SSRI's triggered emergent suicidal ideation above base rates associated with depression." Their citation for this was a 1993 report by the American College of Neuropsychopharmacology. However, in 1987, at an FDA hearing, Tollefson was questioned about a potential link between the SSRIs and suicide, and he did not discuss the fact that the German regulatory body had already raised the issue. In 2003, when asked by the New York Times (August 7) why he did not mention this point at the hearing, he replied: "That was not the question that was asked so I did not answer that question." Yet, textbook chapters are not hearings. They are supposed to be vehicles for the exchange of information among academics; at least that is what many of their readers believe.

While Tollefson and Rosenbaum did not see a correlation between suicidality and SSRI use, apparently others were concerned. In an April 7, 2003 article contrasting statements about concerns with the SSRIs in their internal company documents to the company's public statements exonerating the SSRIs, the New York Times reported:
"Documents brought to light in other cases suggest that Lilly struggled for years to reconcile suicidal events among patients taking Prozac in its trials. One memo shows that a top Lilly executive asked the company's own researchers to record suicide attempts as 'overdose' and thoughts of suicide as "depression.",

Regardless of Tollefson and Rosenbaum's presentation of the suicide issue in 1998, this section of the chapter is now out-of-date as the FDA recently issued a black box warning about a link between suicidal thoughts and the SSRIs. In addition, even Glaxo issued a recent statement warning doctors that there was a six-fold increase in suicidal behavior in young adults taking Paxil. Most importantly, and pertinent to this discussion, as acknowledged by both Glaxo and the FDA, these recent acknowledgments about a link between suicide and the SSRIs were not based on new evidence suddenly coming to light, but were based on a reevaluation of existing evidence.

Apparently, in 1998 there were two different versions of the SSRIs, one being the unpublished version in the pharmaceutical companies' records, and one being the version that was presented to clinicians and scientists in an academic textbook - a textbook published by the American Psychiatric Association, with the first author being an Eli Lilly employee, who said that he would only mention certain data if asked, and the second author being a Lilly consultant.

In addition to the textbook chapter in The American Psychiatric Textbook of Psychopharmacology, the second piece of evidence that Pfizer cited was a review paper titled, The Neurobiology of Depression and Suicide, by Stockmeier. In the first two sentences of the abstract he states: "Alterations in serotonin neurotransmission have been implicated in the pathophysiology of major depression and suicide. However, a picture of serotonergic abnormalities has not emerged from postmortem studies of depression and suicide" (p.220). It is unclear to us how this paper can be used to support Pfizer's position. The paper does mention a pilot study that found an increase in serotonin 1A receptors in the ventrolateral subnucleus of the dorsal raphe nucleus, but it is questionable whether the enormity of the SSRI marketing campaigns can be justified by a 1997 pilot study.

The third piece of evidence for Pfizer was the textbook, Essential Psychopharmacology, by Stahl, containing a very in-depth discussion about the mechanism of action of the SSRIs. When it comes to the discussion about the proof of the chemical imbalance theory, Stahl states: "So far, there is no clear and convincing evidence that monoamine deficiency accounts for depression; that there is no 'real' monoamine deficit." Again, we are not sure how this text supports the chemical imbalance theory, in fact, because this citation refutes Pfizer's position it is a bit strange that they cited it.

As of August 2007, Pfizer's website for Zoloft still contains an explanation of "How Zoloft works" which 
states, "Zoloft works to correct this imbalance of serotonin levels in the brain." An animation on the web page shows how Zoloft works in the brain to accomplish this.

\section{Media Confusion}

Many newspapers and websites continue to mention "chemical imbalances" as if there is an abundance of evidence in the scientific literature. Several authors claimed that there were numerous studies available, yet they did not provide any specifics. One scenario-and it places these omissions in the best possible light — is that the authors are fully aware of the sources of their citations, but simply do not want to spend the time to reply to our requests. Another possibility, however, is that the authors are unable to cite specific citations and their claims are essentially based on hearsay. Either of these scenarios would seem to be an issue for the editors involved, who apparently allowed statements to be made with no supporting evidence. In some cases, we forwarded our dialogue with the reporters to their respective editors. To date, not one of the editors has responded.

Several of the responses from reporters also seem to suggest a fundamental misunderstanding of the chemical imbalance theory as it was originally proposed. For instance, several reporters provided citations that document correlations, but not causation, or they supplied citations that discuss the cellular mechanisms of serotonin binding, which as mentioned before do not document chemical imbalances as a cause for depression.

Yet another possibility is that, for some media outlets, the evidence is just not that important. This scenario presumes that there are two distinct classes of news sources; there are reporters, and there are reporters. In other words, there are media outlets that essentially publish press releases that are handed to them-a press release comes into the office and, with little analysis on the part of the staff, the news bureau simply adds a reporter's name to the byline and republishes the report. These types of media outlets are focused on getting information out to the public in a readable format, while getting the story correct is simply not part of their mission. These sorts of outlets would seem to be more prevalent in the age of the World Wide Web.

On the other hand, there are media outlets that actually investigate, write, and report on an independent basis, and getting the information right is an essential part of their mission. The New York Times would seem to be an example of this second category. Yet, our experience with the Times reporter who discussed the life of Joseph Schildkraut challenges this supposition. While he presented the theory in very matter-of-fact terms, simply stating that the theory had been proved, he has not responded to any of our questions.
A recent newspaper article raises an interesting question: How watered down can the chemical theory become before it is palatable? The market research company, IPSOS, recently conducted a survey of attitudes about mental health and sent out a press release declaring: "Depression in North America: Widespread acknowledgement, understanding." To back up its statement, IPSOS points to the following data: "Virtually all (ninety percent) North Americans believe that depression can [italics added] be caused by a chemical imbalance in the brain." But does the fact that a large group of people believe that depression could be caused by a chemical imbalance warrant the leap to a headline declaring that North Americans understand depression? For a group wishing to promote the theory the headlines certainly sound good, especially as they imply that those who do not subscribe to the chemical imbalance theory must not understand mental illness. Yet, if we look a little closer at the survey's wording, we discover a fundamental flaw. The question IPSOS has asked is virtually meaningless as the vast majority of people, including us, would answer, "Yes." Of course, depression could be caused by a chemical imbalance, but if IPSOS was really interested in finding out if the public understood the chemical imbalance theory then its survey should have asked: Is the belief that depression is caused by a chemical imbalance supported by strong evidence.

Let's look at this wording in another way, removing it from the question of chemical imbalance. We'll use an arbitrary subject - one that has not been proven one way or another. Take the example of extraterrestrials. It is one thing to say: "Extraterrestrials might exist somewhere in the solar system." It is another thing to say, "There is evidence of extraterrestrials in the solar system."

As a side note, it is interesting and possibly not a surprise to see that none of the reports about this survey mentioned that IPSOS, a market research company, handles market development for the pharmaceutical industry.

Our findings also raise an interesting question for the editors of the respective publications: To what extent should reporters be expected to supply references to inquisitive readers requesting more documentation for an article, whether the topic is politics, sports or science? In the world of science, the notion that readers are not entitled to know the evidence in support of an article is unacceptable. Based on the replies we received to our inquiries, in the world of newspaper and magazine publishing, it is apparently considered acceptable to leave readers in the dark.

If the editors in charge of the articles mentioned above had posed the same questions to their own reporters as we had, it is hard to imagine that those editors would have considered the answers acceptable. Whether the answer was: listing a single primary care doctor as their source; citing papers that have nothing to do with the theory; citing doctors who in turn 
would not provide evidence; citing journal supplements; or simply not replying, none of the answers seems adequate. Moreover, in two cases, the authors said they now realized that the discussion was more complicated then they thought, suggesting that a little more editorial oversight would have revealed the problem prior to publication.

\section{Who is Affected by the Reporting?}

An important question surrounding the media's portrayal of the chemical imbalance theory is: What segment of society has this portrayal affected? Certainly, this type of reporting raises issues for the press. A comparison of the media's reporting about mental illness to the biased reporting in the New York Times about the events leading up to the Iraq War does not seem far-fetched. In hindsight, as the Times editors now acknowledge (5/326/04), Judith Miller's war coverage was overly one-sided. Her fundamental flaw could be described as a lack of professional skepticism toward the Bush administration, as she willingly parroted what those pushing for war were saying, while giving little credence to the stance of the other side. Writing in the New York Review of Books, Michael Massing commented that the Times and Miller's reporting were examples of media "submissiveness." This depiction could just as well apply to the media's reporting of mental health issues. As just one example, in some cases, the media still go to the people responsible for the original problems. For instance, several of the researchers involved with the studies of SSRIs in children are still cited in the press even though the following information has come out about their published studies: they downplayed the suicide risk; they exaggerated the benefits; and the papers published under their names were actually written by ghostwriters paid by the pharmaceutical industry. The Times editors have acknowledged both the problems with Miller's reporting and their own lack of editorial oversight of her. It remains to be seen if members of the media will ever look inward and reflect on their role in the promotion of the chemical imbalance theory. (For those familiar with the New York Times' coverage of mental health issues over the past 10 years, it is refreshing that after a series of health reporters who essentially abdicated their role as investigative journalists, there is a newer group of Times reporters with more skeptical inclinations.)

In addition to the press, the other segment of society that would seem to be adversely affected by this reporting consists of the individual psychiatrists and their professional organizations, which have made very few efforts to correct these erroneous claims. Most unfortunately, those who have attempted to do so have been marginalized. For instance, the late Loren Mosher, once the chief of the Center for Studies of Schizophrenia at the National Institute of Mental Health, after years of frustration, in a nowfamous letter, resigned from the American Psychiatry Association (APA), saying, "This is not a group for me. At this point in history, in my view, psychiatry has been almost completely bought out by the drug companies...No longer do we seek to understand whole persons in their social contexts rather we are there to realign our patients' neurotransmitters." For Mosher, the letter was a careerchanging event; his straightforward letter endeared him to many patients but ostracized him from the profession. Mosher, in contrast to the head of the FDA Psychopharmacology Committee who referred to the chemical theory as a "useful metaphor," called the theory a hoax.

When the SSRIs first came on the market the psychiatry profession played an active role in the promotion of the theory, but as evidence has accumulated suggesting that the theory is false, the psychiatry profession has become relatively quiet - they will not defend it, but neither will they take an active role in explaining that it is false. We are not aware of a single psychiatry journal or a related professional organization that has come forward and explained to patients, the media, or professionals about the problematic nature of the advertisements which state that chemical imbalances are the cause of depression. The end result is the party with the most at stake, the patients, have little access to information that would enable them to understand the issue and make a balanced choice. Clearly, a pill makes much more sense if there is really an underlying chemical imbalance. When doctors talk about high blood pressure, diabetes, or high cholesterol they are talking about science. Imagine a patient who has been diagnosed with both high cholesterol and depression talking to his doctor. Given the dearth of reliable information on the subject in the press, the patient can hardly be faulted for not realizing that the discussion about one of his conditions - the high cholesterol - is about science, while the discussion about his other condition - the depression - is about a metaphor. How does the patient know when the discussion has shifted from science to metaphor? For those interested in informed consent this scenario seems problematic.

In closing, it is also important to point out that there are mainstream organizations that accurately portray the chemical imbalance theory, such as the Mental Health Service at McGill University: http:/www.mcgill.ca/mentalhealth/medication/ (accessed May 15, 2007): "The term 'chemical imbalance' is thrown around a lot these days. True conditions caused by chemical imbalances are relatively rare. All thoughts, feelings and motions in the brain are mediated by the release of chemicals in brain pathways. Every person's brain is unique, leading each of us to have different traits and abilities. Just because your brain works in a particular way does not mean that you have a chemical imbalance. A certain amount of sadness, anxiety or other emotional upset is normal, and 
though we may be able to block these feelings by chemicals, this would tend to dehumanize us. Even when we use medication to help an individual with overwhelming emotions, most of the time this is not to repair a "chemical imbalance' but simply to help contain symptoms."

The author of this paragraph has provided an accurate summary of the chemical imbalance theory, although it is at odds with the vast majority of the statements found in the media, the pharmaceutical companies, and NIMH's web page. If patients who have been prescribed antidepressants are provided this information up front, along with information regarding the limited efficacy and potential adverse effects of the medications, this would represent good informed consent. As it is, we are concerned that many patients in clinical encounters are misinformed.

In an earlier paper, we pointed out that to our knowledge; there is not a single peer-reviewed article that can accurately be cited to directly support claims of serotonin deficiency in any mental disorder. Based on our dialogue with the mainstream media, there appears to be no reason to alter this claim. In an effort to continue this conversation, we welcome any replies to the question: What is the evidence that depression is caused by a chemical imbalance?

\section{Further Reading}

Antonuccio, D. et al. (1999). Raising questions about antidepressants. Psychotherapy and Psychosomatics, Vol. 68.

Healy, D. (2006). Let them eat Prozac. New York: New York University Press.
Kirsch, I. et al. (2002). The emperor's new drugs: An analysis of the antidepressant medication data submitted to the US FDA.. Prevention and Treatment. Vol. 5.

Lacasse, J., \& Gomory, T. (2003). Is graduate social work education promoting a critical approach to mental health practice? Journal of Social Work Education, 39(3), 383-408.

Lacasse, J.R. and Leo, J. (2005). Serotonin and depression: A disconnect between the advertisements and the scientific literature. PLoS Med 2(12): e392. Available at: http://dx.doi.org/10.1371/ journal.pmed.0020392.

Lacasse, J. and Leo, J. (2006). Serotonin and depression: A disconnect between the advertisements and the scientific literature. PLoS Medicine. Available at: http://medicine.plosjournals.org/perlserv/ ?request=get-document\&doi=10.1371/journal.pmed.0020392.

Leo, J. (2006). The SSRI trials in children. Ethical Human Psychiatry and Psychology, 8(1), 29-41.

Jonathan Leo, Ph.D. is an Associate Professor of Neuroanatomy at Lincoln Memorial University in Harrogate TN. He has published numerous articles about mental health covering topics such as the genetic basis of schizophrenia, the serotonin theory of depression, and the pediatric trials of SSRIs. For further questions he can be reached at jonathan.leo@lmunet.edu.

Jeffrey R. Lacasse, MSW is a Visiting Lecturer at the Florida State University (FSU). He has published research on practice with children, critical thinking in graduate social work education, and consumer advertising of SSRI antidepressants. He can be reached at jeffreylacasse@mac.com. 